\title{
Norms over bifuzzy bi-ideals with operators in semigroups
}

\author{
Rasul Rasuli \\ Department of Mathematics, Payame Noor University \\ Tehran, Iran \\ e-mail: rasulirasul@yahoo.com
}

Received: 23 July 2017

Revised: 23 March 2019

Accepted: 1 April 2019

\begin{abstract}
In this paper, by using norms ( $T$ and $C$ ) we introduce the concepts of $\Omega$-bifuzzy subsemigroups, $\Omega$-bifuzzy ideals and $\Omega$-bifuzzy bi-ideals of semigroup $S$ and consider some of their properties and structured characteristics.
\end{abstract}

Keywords: Theory of groups, Ideals, Norms, Intuitionistic mathematics, Fuzzy set theory, Lattice.

2010 Mathematics Subject Classification: 05B10, 06B10, 03B45, 03F55, 03E72, 06D50.

\section{Introduction}

In mathematics, fuzzy sets are sets whose elements have degrees of membership. Fuzzy sets were introduced by Lotfi A. Zadeh[25] in 1965 as an extension of the classical notion of set and after that D. Klaua investigated some properties of fuzzy sets ([10]). In classical set theory, the membership of elements in a set is assessed in binary terms according to a bivalent condition an element either belongs or does not belong to the set. By contrast, fuzzy set theory permits the gradual assessment of the membership of elements in a set; this is described with the aid of a membership function valued in the real unit interval $[0,1]$. Fuzzy sets generalize classical sets, since the indicator functions of classical sets are special cases of the membership functions of fuzzy sets, if the latter only take values 0 or 1 (see [5]). In fuzzy set theory, classical bivalent sets are usually called crisp sets. The fuzzy set theory can be used in a wide range of domains in 
which information is incomplete or imprecise, such as bioinformatics (see [16]). As a generalization of a fuzzy set, the concept of an intuitionistic fuzzy set was introduced by K. T. Atanassov $[2,3]$. In mathematics, a join-semilattice (or upper semilattice) is a partially ordered set that has a join (a least upper bound) for any nonempty finite subset. Dually, a meet-semilattice (or lower semilattice) is a partially ordered set which has a meet (or greatest lower bound) for any nonempty finite subset. Every join-semilattice is a meet-semilattice in the inverse order and vice versa. Semilattices can also be defined algebraically: join and meet are associative, commutative, idempotent binary operations, and any such operation induces a partial order (and the respective inverse order) such that the result of the operation for any two elements is the least upper bound (or greatest lower bound) of the elements with respect to this partial order. A lattice is a partially ordered set that is both a meet- and join-semilattice with respect to the same partial order. Algebraically, a lattice is a set with two associative, commutative idempotent binary operations linked by corresponding absorption laws (see [1]). In mathematics, a $t$-norm (also $T$-norm or, unabbreviated, triangular norm) is a kind of binary operation used in the framework of probabilistic metric spaces and in multi-valued logic, specifically in fuzzy logic. A $t$-norm generalizes intersection in a lattice and conjunction in logic. The name triangular norm refers to the fact that in the framework of probabilistic metric spaces $t$-norms are used to generalize triangle inequality of ordinary metric spaces. In [11, 12], Kuroki have studied several properties of fuzzy left (right) ideals, fuzzy bi-ideals and fuzzy interior ideals in semigroups. For more other study on the fuzzy theory in semigroups, we refer to papers [7, 13, 17, 23, 24]. Hur et al. [9] have studied the bifuzzy structure of subsemigroups with operators in semigroups. The author by using norms, investigated some properties of fuzzy submodules, fuzzy subrings, fuzzy ideals of subtraction semigroups, intuitionistic fuzzy subrings and ideals of a ring (see [20, 21, 19, 22]). The purpose of this paper is to deal with the algebraic structure of $\Omega$-bifuzzy subsemigroups, $\Omega$-bifuzzy ideals and $\Omega$-bifuzzy bi-ideals of semigroup $S$ under norms. In Section 2, we summarize some basic concepts which will be used throughout the paper. In Section 3, by using norms, we redefined the concepts of $\Omega$-bifuzzy subsemigroups, $\Omega$-bifuzzy ideals and $\Omega$-bifuzzy bi-ideals of semigroup $S$ and under some conditions for $S$ (as regular, completely regular, semilattice) will give some related properties and new results.

\section{Preliminaries}

In this section, we recall some of the fundamental concepts and definition, which are necessary for this paper.

Definition 2.1. (see [8]) Let $(S, *)$ be a mathematical system such that $(a * b) * c=a *(b * c)$ for all $a, b, c \in S$. Then $*$ is called a ssociative and $(S, *)$ is called a semigroup.

Definition 2.2. (see [8])

(1) A semigroup $S$ is called regular if for all $a \in S$, there exists $x \in S$ such that $a=a x a$.

(2) A semigroup $S$ is called completely regular if for all $a \in S$, there exists $x \in S$ such that 
$a=a x a$ and $a x=x a$.

(3) By a subsemigroup of a semigroup $S$ we mean a nonempty subset $A$ of $S$ such that $A^{2} \subseteq A$, and by a left (right) ideal of $S$ we mean a non-empty subset $A$ of $S$ such that $S A \subseteq A(A S \subseteq A)$. By an ideal, we mean a nonempty subset of a semigroup $S$ which is both a left and a right ideal of $S$. A subsemigroup $A$ of a semigroup $S$ is called a bi-ideal of $S$ if $A S A \subseteq A$.

Definition 2.3. (see [14]) A nonempty subset $A$ of a semigroup $S$ is called a generalized bi-ideal of $S$ if $A S A \subseteq A$.

Definition 2.4. Let $X$ a non-empty sets. A fuzzy subset $\mu$ of $X$ is a function $\mu: X \rightarrow[0,1]$. Denote by $[0,1]^{X}$, the set of all fuzzy subset of $X$.

Definition 2.5. (see [4]) A $t$-norm $T$ is a function $T:[0,1] \times[0,1] \rightarrow[0,1]$ having the following four properties:

(T1) $T(x, 1)=x$ (neutral element),

(T2) $T(x, y) \leq T(x, z)$ if $y \leq z$ (monotonicity),

(T3) $T(x, y)=T(y, x)$ (commutativity),

(T4) $T(x, T(y, z))=T(T(x, y), z)$ (associativity),

for all $x, y, z \in[0,1]$.

It is clear that if $x_{1} \geq x_{2}$ and $y_{1} \geq y_{2}$, then $T\left(x_{1}, y_{1}\right) \geq T\left(x_{2}, y_{2}\right)$.

Example 2.6. (1) Standard intersection $T$-norm $T_{m}(x, y)=\min \{x, y\}$.

(2) Bounded sum $T$-norm $T_{b}(x, y)=\max \{0, x+y-1\}$.

(3) algebraic product $T$-norm $T_{p}(x, y)=x y$.

(4) Drastic $T$-norm

$$
T_{D}(x, y)= \begin{cases}y, & \text { if } x=1 \\ x, & \text { if } y=1 \\ 0, & \text { otherwise. }\end{cases}
$$

(5) Nilpotent minimum $T$-norm

$$
T_{n M}(x, y)=\left\{\begin{aligned}
\min \{x, y\}, & \text { if } x+y>1 \\
0, & \text { otherwise }
\end{aligned}\right.
$$

(6) Hamacher product $T$-norm

$$
T_{H_{0}}(x, y)=\left\{\begin{aligned}
0, & \text { if } x=y=0 \\
\frac{x y}{x+y-x y}, & \text { otherwise. }
\end{aligned}\right.
$$

The drastic $t$-norm is the pointwise smallest $t$-norm and the minimum is the pointwise largest $t$-norm: $T_{D}(x, y) \leq T(x, y) \leq T_{\min }(x, y)$ for all $x, y \in[0,1]$. 
Definition 2.7. (see [4]) A $t$-conorm $C$ is a function $C:[0,1] \times[0,1] \rightarrow[0,1]$ having the following four properties:

(C1) $C(x, 0)=x$,

(C2) $C(x, y) \leq C(x, z)$ if $y \leq z$,

(C3) $C(x, y)=C(y, x)$,

(C4) $C(x, C(y, z))=C(C(x, y), z)$,

for all $x, y, z \in[0,1]$.

Example 2.8. (1) Standard union $t$-conorm $C_{m}(x, y)=\max \{x, y\}$.

(2) Bounded sum $t$-conorm $C_{b}(x, y)=\min \{1, x+y\}$.

(3) Algebraic sum $t$-conorm $C_{p}(x, y)=x+y-x y$.

(4) Drastic $T$-conorm

$$
C_{D}(x, y)= \begin{cases}y, & \text { if } x=0 \\ x, & \text { if } y=0 \\ 1, & \text { otherwise }\end{cases}
$$

dual to the drastic $T$-norm.

(5) Nilpotent maximum $T$-conorm, dual to the nilpotent minimum $T$-norm:

$$
C_{n M}(x, y)=\left\{\begin{aligned}
\max \{x, y\}, & \text { if } x+y<1 \\
1, & \text { otherwise. }
\end{aligned}\right.
$$

(6) Einstein sum (compare the velocity-addition formula under special relativity) $C_{H_{2}}(x, y)=$ $\frac{x+y}{1+x y}$ is a dual to one of the Hamacher $t$-norms. Note that all $t$-conorms are bounded by the maximum and the drastic t-conorm: $C_{\max }(x, y) \leq C(x, y) \leq C_{D}(x, y)$ for any $t$-conorm $C$ and all $x, y \in[0,1]$.

Recall that $t$-norm $T$ (respectively, $t$-conorm $C$ ) is idempotent if for all $x \in[0,1], T(x, x)=x$ (respectively, $C(x, x)=x$ ).

Definition 2.9. (see [19]) Let $\mu \in[0,1]^{S}$ and $x, y \in S$. We say that $\mu$ is a fuzzy subsemigroup of $S$ with respect to a $t$-norm $T$ if $\mu(x y) \geq T(\mu(x), \mu(y))$.

Definition 2.10. (see [19]) A fuzzy set $\mu$ in semigroup $S$ is called a fuzzy ideal of $S$ with respect to a $t$-norm $T$ if it satisfies the following axioms:

(1) $\mu(x y) \geq T(\mu(x), \mu(y))$,

(2) $\mu(x y) \geq \mu(y)$,

(3) $\mu(x y) \geq \mu(x)$

for all $x, y \in S$.

Definition 2.11. (see [19]) A fuzzy set $\mu$ in semigroup $S$ is called a fuzzy bi-ideal of $S$ with respect to a $t$-norm $T$ if it satisfies the following axioms:

(1) $\mu(x y) \geq T(\mu(x), \mu(y))$,

(2) $\mu(x w y) \geq T(\mu(x), \mu(y))$

for all $x, w, y \in S$. 
Definition 2.12. (see $[2,6]$ ) Let $X$ be a nonempty set. An intuitionistic fuzzy set is an object of the form: $A=\left\{\left(x, \mu_{A}(x), \nu_{A}(x)\right): x \in X\right\}$ such that $\mu_{A}, \nu_{A} \in[0,1]^{X}$ and for all $x \in X$ we have $\left(\mu_{A}(x)+\nu_{A}(x)\right) \in[0,1]$. This object is also called a bifuzzy set. For the sake of simplicity, we shall use the symbol $A=\left(X, \mu_{A}, \nu_{A}\right)$ for the $A=\left\{\left(x, \mu_{A}(x), \nu_{A}(x)\right): x \in X\right\}$.

Definition 2.13. (see [19]) A bifuzzy set $A=\left(S, \mu_{A}, \nu_{A}\right)$ in a semigroup $S$ is called bifuzzy subsemigroup of $S$ with respect to a $t$-norm $T$ and a $t$-conorm $C$, if it satisfies:

(1) $\mu_{A}(x y) \geq T\left(\mu_{A}(x), \mu_{A}(y)\right)$,

(2) $\nu_{A}(x y) \leq C\left(\nu_{A}(x), \nu_{A}(y)\right)$

for all $x, y \in S$.

Definition 2.14. (see [19]) A bifuzzy set $A=\left(S, \mu_{A}, \nu_{A}\right)$ in a semigroup $S$ is called bifuzzy ideal of $S$ with respect to a $t$-norm $T$ and a $t$-conorm $C$, if it satisfies:

(1) $\mu_{A}(x y) \geq T\left(\mu_{A}(x), \mu_{A}(y)\right)$,

(2) $\mu_{A}(x y) \geq \mu_{A}(x)$,

(3) $\mu_{A}(x y) \geq \mu_{A}(y)$,

(4) $\nu_{A}(x y) \leq C\left(\nu_{A}(x), \nu_{A}(y)\right)$,

(5) $\nu_{A}(x y) \leq \nu_{A}(y)$,

(6) $\nu_{A}(x y) \leq \nu_{A}(x)$

for all $x, y \in S$.

Definition 2.15. (see [19]) A bifuzzy set $A=\left(S, \mu_{A}, \nu_{A}\right)$ in a semigroup $S$ is called bifuzzy bi-ideal of $S$ with respect to a $t$-norm $T$ and a $t$-conorm $C$, if it satisfies:

(1) $\mu_{A}(x y) \geq T\left(\mu_{A}(x), \mu_{A}(y)\right)$,

(2) $\mu_{A}(x w y) \geq T\left(\mu_{A}(x), \mu_{A}(y)\right)$,

(3) $\nu_{A}(x y) \leq C\left(\nu_{A}(x), \nu_{A}(y)\right)$,

(4) $\nu_{A}(x w y) \leq C\left(\nu_{A}(x), \nu_{A}(y)\right)$

for all $x, w, y \in S$.

Lemma 2.16. (see [18]) For a semigroup $S$ the following conditions are equivalent:

(1) $S$ is completely regular.

(2) $S$ is a union of groups.

(3) $a \in a^{2} S a^{2}$ for all $a \in S$.

Lemma 2.17. (see [15]) A semigroup $S$ is a semilattice of groups if and only if the set of all bi-ideals of $S$ is a semilattice under the multiplication of subsets.

\section{Norms over $\Omega$-bifuzzy bi-ideals}

In what follows let $S$ and $\Omega$ denote a semigroup and a nonempty set, respectively, unless otherwise specified. 
Definition 3.1. An $\Omega$-bifuzzy set $A_{\Omega}$ in $S$ is defined to be an object having the form

$$
A_{\Omega}=\left\{\left((x, \alpha), \mu_{A_{\Omega}}(x, \alpha), \nu_{A_{\Omega}}(x, \alpha)\right):(x, \alpha) \in S \times \Omega\right\},
$$

where the function $\mu_{A_{\Omega}}: S \times \Omega \rightarrow[0,1]$ and $\nu_{A_{\Omega}}: S \times \Omega \rightarrow[0,1]$ define the degree of membership and the degree of nonmembership of the element $(x, \alpha) \in S \times \Omega$ to $A_{\Omega} \subset S \times \Omega$, respectively, such that for all $(x, \alpha) \in S \times \Omega$ we have that $\left(\mu_{A_{\Omega}}(x, \alpha)+\nu_{A_{\Omega}}(x, \alpha)\right) \in[0,1]$. We shall use the symbol $A_{\Omega}=\left(S \times \Omega, \mu_{A_{\Omega}}, \nu_{A_{\Omega}}\right)$ for the $\Omega$-bifuzzy set

$$
A_{\Omega}=\left\{\left((x, \alpha), \mu_{A_{\Omega}}(x, \alpha), \nu_{A_{\Omega}}(x, \alpha)\right):(x, \alpha) \in S \times \Omega\right\} .
$$

Definition 3.2. An $\Omega$-bifuzzy $A_{\Omega}=\left(S \times \Omega, \mu_{A_{\Omega}}, \nu_{A_{\Omega}}\right)$ in $S$ is called an $\Omega$-bifuzzy subsemigroup of $S$ under norms ( $T$ and $C$ ), if it satisfies:

(1) $\mu_{A_{\Omega}}(x y, \alpha) \geq T\left(\mu_{A_{\Omega}}(x, \alpha), \mu_{A_{\Omega}}(y, \alpha)\right)$,

(2) $\nu_{A_{\Omega}}(x y, \alpha) \leq C\left(\nu_{A_{\Omega}}(x, \alpha), \nu_{A_{\Omega}}(y, \alpha)\right)$,

for all $x, y \in S$ and $\alpha \in \Omega$.

Definition 3.3. An $\Omega$-bifuzzy $A_{\Omega}=\left(S \times \Omega, \mu_{A_{\Omega}}, \nu_{A_{\Omega}}\right)$ in $S$ is called an $\Omega$-bifuzzy ideal of $S$ under norms ( $T$ and $C$ ), if it satisfies:

(1) $\mu_{A_{\Omega}}(x y, \alpha) \geq T\left(\mu_{A_{\Omega}}(x, \alpha), \mu_{A_{\Omega}}(y, \alpha)\right)$,

(2) $\mu_{A_{\Omega}}(x y, \alpha) \geq \mu_{A_{\Omega}}(x, \alpha)$,

(3) $\mu_{A_{\Omega}}(x y, \alpha) \geq \mu_{A_{\Omega}}(x, \alpha)$,

(4) $\nu_{A_{\Omega}}(x y, \alpha) \leq C\left(\nu_{A_{\Omega}}(x, \alpha), \nu_{A_{\Omega}}(y, \alpha)\right)$,

(5) $\nu_{A_{\Omega}}(x y, \alpha) \leq \nu_{A_{\Omega}}(y, \alpha)$,

(6) $\nu_{A_{\Omega}}(x y, \alpha) \leq \nu_{A_{\Omega}}(x, \alpha)$

for all $x, y \in S$ and $\alpha \in \Omega$.

Lemma 3.4. Let $S^{\Omega}:=\{u \mid u: \Omega \rightarrow S\}$ such that for all $u, v \in S^{\Omega}$ and $\alpha \in \Omega$ we define $(u v)(\alpha)=u(\alpha) v(\alpha)$. Then $S^{\Omega}$ is a semigroup.

Proof. Let $u, v \in S^{\Omega}$ and $\alpha \in \Omega$. Then $(u v)(\alpha)=u(\alpha) v(\alpha) \in S$ (as $S$ is a semigroup) and so $S^{\Omega}$ is a semigroup.

Lemma 3.5. Let $A_{\Omega}=\left(S \times \Omega, \mu_{A_{\Omega}}, \nu_{A_{\Omega}}\right)$ be an $\Omega$-bifuzzy subsemigroup of $S$ under norms ( $T$ and $C)$. Define bifuzzy set $\Phi=\left(S^{\Omega}, \mu_{\Phi}, \nu_{\Phi}\right)$ in $S^{\Omega}$ by $\mu_{\Phi}(u)=\inf \left\{\mu_{A_{\Omega}}(u(\alpha), \alpha) \mid \alpha \in \Omega\right\}$ and $\nu_{\Phi}(u)=\sup \left\{\nu_{A_{\Omega}}(u(\alpha), \alpha) \mid \alpha \in \Omega\right\}$ such that $u \in S^{\Omega}$. Then $\Phi=\left(S^{\Omega}, \mu_{\Phi}, \nu_{\Phi}\right)$ is a bifuzzy subsemigroup of $S^{\Omega}$ under norms ( $T$ and $C$ ).

Proof. Let $u, v \in S^{\Omega}$. Then

$$
\begin{gathered}
\mu_{\Phi}(u v)=\inf \left\{\mu_{A_{\Omega}}((u v)(\alpha), \alpha) \mid \alpha \in \Omega\right\}=\inf \left\{\mu_{A_{\Omega}}(u(\alpha) v(\alpha), \alpha) \mid \alpha \in \Omega\right\} \\
\geq \inf \left\{T\left(\mu_{A_{\Omega}}(u(\alpha), \alpha), \mu_{A_{\Omega}}(v(\alpha), \alpha)\right) \mid \alpha \in \Omega\right\} \\
=T\left(\inf \left\{\mu_{A_{\Omega}}(u(\alpha), \alpha) \mid \alpha \in \Omega\right\}, \inf \left\{\mu_{A_{\Omega}}(v(\alpha), \alpha) \mid \alpha \in \Omega\right\}\right) \\
=T\left(\mu_{\Phi}(u), \mu_{\Phi}(v)\right) .
\end{gathered}
$$


Also

$$
\begin{gathered}
\nu_{\Phi}(u v)=\sup \left\{\nu_{A_{\Omega}}((u v)(\alpha), \alpha) \mid \alpha \in \Omega\right\}=\sup \left\{\nu_{A_{\Omega}}(u(\alpha) v(\alpha), \alpha) \mid \alpha \in \Omega\right\} \\
\leq \sup \left\{C\left(\nu_{A_{\Omega}}(u(\alpha), \alpha), \nu_{A_{\Omega}}(v(\alpha), \alpha)\right) \mid \alpha \in \Omega\right\} \\
=C\left(\sup \left\{\nu_{A_{\Omega}}(u(\alpha), \alpha) \mid \alpha \in \Omega\right\}, \sup \left\{\nu_{A_{\Omega}}(v(\alpha), \alpha) \mid \alpha \in \Omega\right\}\right) \\
=C\left(\nu_{\Phi}(u), \nu_{\Phi}(v)\right) .
\end{gathered}
$$

Thus $\Phi=\left(S^{\Omega}, \mu_{\Phi}, \nu_{\Phi}\right)$ will be a bifuzzy subsemigroup of $S^{\Omega}$ under norms ( $T$ and $C$ ).

Proposition 3.6. Let $A_{\Omega}=\left(S \times \Omega, \mu_{A_{\Omega}}, \nu_{A_{\Omega}}\right)$ be an $\Omega$-bifuzzy ideal of Sunder norms ( $T$ and $C$ ). Then $\Phi=\left(S^{\Omega}, \mu_{\Phi}, \nu_{\Phi}\right)$ given in Lemma 3.5 will be a bifuzzy ideal of $S^{\Omega}$ under norms ( $T$ and $C$ ).

Proof. Let $u, v \in S^{\Omega}$ then from Lemma 3.5 we get that $\mu_{\Phi}(u v) \geq T\left(\mu_{\Phi}(u), \mu_{\Phi}(v)\right)$ and $\nu_{\Phi}(u v) \leq C\left(\mu_{\Phi}(u), \mu_{\Phi}(v)\right)$. Now

$$
\begin{gathered}
\mu_{\Phi}(u v)=\inf \left\{\mu_{A_{\Omega}}((u v)(\alpha), \alpha) \mid \alpha \in \Omega\right\}=\inf \left\{\mu_{A_{\Omega}}(u(\alpha) v(\alpha), \alpha) \mid \alpha \in \Omega\right\} \\
\geq \inf \left\{\mu_{A_{\Omega}}(u(\alpha), \alpha) \mid \alpha \in \Omega\right\}=\mu_{\Phi}(u) .
\end{gathered}
$$

Also

$$
\begin{gathered}
\nu_{\Phi}(u v)=\sup \left\{\nu_{A_{\Omega}}((u v)(\alpha), \alpha) \mid \alpha \in \Omega\right\}=\sup \left\{\nu_{A_{\Omega}}(u(\alpha) v(\alpha), \alpha) \mid \alpha \in \Omega\right\} \\
\leq \sup \left\{\nu_{A_{\Omega}}(u(\alpha), \alpha) \mid \alpha \in \Omega\right\}=\nu_{\Phi}(u) .
\end{gathered}
$$

Similarly $\mu_{\Phi}(u v) \geq \mu_{\Phi}(v)$ and $\nu_{\Phi}(u v) \leq \nu_{\Phi}(v)$. Therefore $\Phi=\left(S^{\Omega}, \mu_{\Phi}, \nu_{\Phi}\right)$ is a bifuzzy ideal of $S^{\Omega}$ under norms ( $T$ and $C$ ).

Definition 3.7. An $\Omega$-bifuzzy set $A_{\Omega}=\left(S \times \Omega, \mu_{A_{\Omega}}, \nu_{A_{\Omega}}\right)$ in $S$ is called an $\Omega$-bifuzzy bi-ideal of $S$ under norms ( $T$ and $C$ ), if it satisfying the following assertions:

(1) $\mu_{A_{\Omega}}(x y, \alpha) \geq T\left(\mu_{A_{\Omega}}(x, \alpha), \mu_{A_{\Omega}}(y, \alpha)\right)$,

(2) $\mu_{A_{\Omega}}(x w y, \alpha) \geq T\left(\mu_{A_{\Omega}}(x, \alpha), \mu_{A_{\Omega}}(y, \alpha)\right)$,

(3) $\nu_{A_{\Omega}}(x y, \alpha) \leq C\left(\nu_{A_{\Omega}}(x, \alpha), \nu_{A_{\Omega}}(y, \alpha)\right)$,

(4) $\nu_{A_{\Omega}}(x w y, \alpha) \leq C\left(\nu_{A_{\Omega}}(x, \alpha), \nu_{A_{\Omega}}(y, \alpha)\right)$

for all $x, w, y \in S$ and $\alpha \in \Omega$.

Example 3.8. Let $A=\left(S, \mu_{A}, \nu_{A}\right)$ be a bifuzzy set in $S$ and let $A_{\Omega}=\left(S^{\Omega} \times \Omega, \mu_{A_{\Omega}}, \nu_{A_{\Omega}}\right)$ be an $\Omega$-bifuzzy set in $S^{\Omega}$ defined by $\mu_{A_{\Omega}}(u, \alpha)=\mu_{A}(u(\alpha))$ and $\nu_{A_{\Omega}}(u, \alpha)=\nu_{A}(u(\alpha))$ for all $u \in S^{\Omega}$ and $\alpha \in \Omega$.

(1) If $A=\left(S, \mu_{A}, \nu_{A}\right)$ be a bifuzzy subsemigroup of $S$ under norms ( $T$ and $C$ ), then $A_{\Omega}=$ $\left(S^{\Omega} \times \Omega, \mu_{A_{\Omega}}, \nu_{A_{\Omega}}\right)$ be an $\Omega$-bifuzzy subsemigroup of $S^{\Omega}$ under norms ( $T$ and $C$ ).

(2) If $A=\left(S, \mu_{A}, \nu_{A}\right)$ be a bifuzzy bi-ideal of $S$ under norms ( $T$ and $\left.C\right)$, then $A_{\Omega}=\left(S^{\Omega} \times\right.$ $\left.\Omega, \mu_{A_{\Omega}}, \nu_{A_{\Omega}}\right)$ be an $\Omega$-bifuzzy bi-ideal of $S^{\Omega}$ under norms ( $T$ and $C$ ).

Example 3.9. Let $\Omega:=\left\{A=\left(S, \mu_{A}, \nu_{A}\right) \mid A\right.$ is a bifuzzy bi-ideal of $S$ under norms $(T$ and $\left.C)\right\}$. Define $\Psi=\left(S \times \Omega, \mu_{\Psi}, \nu_{\Psi}\right)$ as an $\Omega$-bifuzzy set in $S$ by $\mu_{\Psi}\left(x, \mu_{A}\right)=\mu_{A}(x)$ and $\nu_{\Psi}\left(x, \mu_{A}\right)=$ $\nu_{A}(x)$ for all $x \in S$ and $A \in \Omega$. Then $\Psi$ is an $\Omega$-bifuzzy bi-ideal of $S$ under norms ( $T$ and $C$ ). 
Example 3.10. Let $S=\{a, b\}$ be a semigroup with the following Cayley table:

\begin{tabular}{c|cc} 
& $a$ & $b$ \\
\hline$a$ & $a$ & $b$ \\
$b$ & $b$ & $a$
\end{tabular}

also let $\Omega=\{1,2,3\}$ and $A_{\Omega}=\left(S \times \Omega, \mu_{A_{\Omega}}, \nu_{A_{\Omega}}\right)$ be an $\Omega$-bifuzzy set in $S$ defined by

$$
\begin{aligned}
A_{\Omega}=(S \times \Omega, & \left\{\frac{(a, 1)}{1}, \frac{(a, 2)}{1}, \frac{(a, 3)}{1}, \frac{(b, 1)}{0.9}, \frac{(b, 2)}{0.8}, \frac{(b, 3)}{0.7}\right\}, \\
& \left.\left\{\frac{(a, 1)}{0}, \frac{(a, 2)}{0}, \frac{(a, 3)}{0}, \frac{(b, 1)}{0.2}, \frac{(b, 2)}{0.3}, \frac{(b, 3)}{0.4}\right\}\right) .
\end{aligned}
$$

Then $A_{\Omega}$ is an $\Omega$-bifuzzy subsemigroup of $S$ under norms $\left(T(x, y)=T_{m}(x, y)=\min \{x, y\}\right.$ and $\left.C(x, y)=C_{m}(x, y)=\max \{x, y\}\right)$ for all $x, y \in[0,1]$. Since $\mu_{A_{\Omega}}(a b a, 3)=\mu_{A_{\Omega}}(b, 3)=$ $0.7 \ngtr T\left(\mu_{A_{\Omega}}(a, 3), \mu_{A_{\Omega}}(a, 3)\right)=\max \{1,1\}=1$, and $\nu_{A_{\Omega}}(a b a, 2)=\nu_{A_{\Omega}}(b, 2)=0.3$ $\left.C\left(\nu_{A_{\Omega}}(a, 2), \nu_{A_{\Omega}}\right)(a, 2)\right)=\max \{0,0\}=0$, so $A_{\Omega}$ is not an $\Omega$-bifuzzy bi-ideal of $S$ under norms $\left(T(x, y)=T_{m}(x, y)=\min \{x, y\}\right.$ and $\left.C(x, y)=C_{m}(x, y)=\max \{x, y\}\right)$ for all $x, y \in[0,1]$.

Theorem 3.11. Let $A_{\Omega}=\left(S \times \Omega, \mu_{A_{\Omega}}, \nu_{A_{\Omega}}\right)$ be an $\Omega$-bifuzzy set in $S$. Define a bifuzzy set $A_{\Omega}^{\alpha}=\left(S, \mu_{A_{\Omega}^{\alpha}}, \nu_{A_{\Omega}^{\alpha}}\right)$ in $S$ by $\mu_{A_{\Omega}^{\alpha}}(x)=\mu_{A_{\Omega}}(x, \alpha)$ and $\nu_{A_{\Omega}^{\alpha}}(x)=\nu_{A_{\Omega}}(x, \alpha)$ for all $\alpha \in \Omega, x \in S$. Then $A_{\Omega}^{\alpha}$ is a bifuzzy bi-ideal of $S$ under norms ( $T$ and $C$ ) if and only if $A_{\Omega}$ is an $\Omega$-bifuzzy bi-ideal of $S$ under norms ( $T$ and $C$ ).

Proof. Let $x, w, y \in S$ and $\alpha \in \Omega$.

If $A_{\Omega}^{\alpha}$ is a bifuzzy bi-ideal of $S$ under norms ( $T$ and $C$ ), then

(1) $\mu_{A_{\Omega}}(x y, \alpha)=\mu_{A_{\Omega}^{\alpha}}(x y) \geq T\left(\mu_{A_{\Omega}^{\alpha}}(x), \mu_{A_{\Omega}^{\alpha}}(y)\right)=T\left(\mu_{A_{\Omega}}(x, \alpha), \mu_{A_{\Omega}}(y, \alpha)\right)$.

(2) $\mu_{A_{\Omega}}(x w y, \alpha)=\mu_{A_{\Omega}^{\alpha}}(x w y) \geq T\left(\mu_{A_{\Omega}^{\alpha}}(x), \mu_{A_{\Omega}^{\alpha}}(y)\right)=T\left(\mu_{A_{\Omega}}(x, \alpha), \mu_{A_{\Omega}}(y, \alpha)\right)$.

(3) $\nu_{A_{\Omega}}(x y, \alpha)=\nu_{A_{\Omega}^{\alpha}}(x y) \leq C\left(\nu_{A_{\Omega}^{\alpha}}(x), \nu_{A_{\Omega}^{\alpha}}(y)\right)=C\left(\nu_{A_{\Omega}}(x, \alpha), \nu_{A_{\Omega}}(y, \alpha)\right)$.

(4) $\nu_{A_{\Omega}}(x w y, \alpha)=\nu_{A_{\Omega}^{\alpha}}(x w y) \leq C\left(\nu_{A_{\Omega}^{\alpha}}(x), \nu_{A_{\Omega}^{\alpha}}(y)\right)=C\left(\nu_{A_{\Omega}}(x, \alpha), \nu_{A_{\Omega}}(y, \alpha)\right)$.

Thus $A_{\Omega}$ is an $\Omega$-bifuzzy bi-ideal of $S$ under norms ( $T$ and $C$ ). Conversely, let $A_{\Omega}$ is an $\Omega$-bifuzzy bi-ideal of $S$ under norms ( $T$ and $C$ ). Then

(1) $\mu_{A_{\Omega}^{\alpha}}(x y)=\mu_{A_{\Omega}}(x y, \alpha) \geq T\left(\mu_{A_{\Omega}}(x, \alpha), \mu_{A_{\Omega}}(y, \alpha)\right)=T\left(\mu_{A_{\Omega}^{\alpha}}(x), \mu_{A_{\Omega}^{\alpha}}(y)\right)$.

(2) $\mu_{A_{\Omega}^{\alpha}}(x w y)=\mu_{A_{\Omega}}(x w y, \alpha) \geq T\left(\mu_{A_{\Omega}}(x, \alpha), \mu_{A_{\Omega}}(y, \alpha)\right)=T\left(\mu_{A_{\Omega}^{\alpha}}(x), \mu_{A_{\Omega}^{\alpha}}(y)\right)$.

(3) $\nu_{A_{\Omega}^{\alpha}}(x y)=\nu_{A_{\Omega}}(x y, \alpha) \leq C\left(\nu_{A_{\Omega}}(x, \alpha), \nu_{A_{\Omega}}(y, \alpha)\right)=C\left(\nu_{A_{\Omega}^{\alpha}}(x), \nu_{A_{\Omega}^{\alpha}}(y)\right)$.

(4) $\nu_{A_{\Omega}^{\alpha}}(x w y)=\nu_{A_{\Omega}}(x w y, \alpha) \leq C\left(\nu_{A_{\Omega}}(x, \alpha), \nu_{A_{\Omega}}(y, \alpha)\right)=C\left(\nu_{A_{\Omega}^{\alpha}}(x), \nu_{A_{\Omega}^{\alpha}}(y)\right)$.

Therefore, $A_{\Omega}^{\alpha}$ is a bifuzzy bi-ideal of $S$ under norms ( $T$ and $C$ ).

Proposition 3.12. Let $S$ be a group. If $T$ and $C$ are idempotent, then every $\Omega$-bifuzzy bi-ideal of $S$ under norms ( $T$ and $C$ ) will be a constant function.

Proof. Let $A_{\Omega}=\left(S \times \Omega, \mu_{A_{\Omega}}, \nu_{A_{\Omega}}\right)$ is an $\Omega$-bifuzzy bi-ideal of $S$ under norms ( $T$ and $C$ ) and $e$ is the identity element of $S$. Then for all $x \in S$ and $\alpha \in \Omega$ we obtain that

$$
\mu_{A_{\Omega}}(x, \alpha)=\mu_{A_{\Omega}}(\text { exe, } \alpha) \geq T\left(\mu_{A_{\Omega}}(e, \alpha), \mu_{A_{\Omega}}(e, \alpha)\right)=\mu_{A_{\Omega}}(e, \alpha)
$$




$$
\begin{gathered}
=\mu_{A_{\Omega}}(e e, \alpha)=\mu_{A_{\Omega}}\left(\left(x x^{-1}\right)\left(x^{-1} x\right), \alpha\right)=\mu_{A_{\Omega}}\left(x\left(x^{-1} x^{-1}\right) x, \alpha\right) \\
\geq T\left(\mu_{A_{\Omega}}(x, \alpha), \mu_{A_{\Omega}}(x, \alpha)\right)=\mu_{A_{\Omega}}(x, \alpha) .
\end{gathered}
$$

Thus $\mu_{A_{\Omega}}(x, \alpha)=\mu_{A_{\Omega}}(e, \alpha)$. Also

$$
\begin{gathered}
\nu_{A_{\Omega}}(x, \alpha)=\nu_{A_{\Omega}}(e x e, \alpha) \leq C\left(\nu_{A_{\Omega}}(e, \alpha), \nu_{A_{\Omega}}(e, \alpha)\right)=\nu_{A_{\Omega}}(e, \alpha) \\
=\nu_{A_{\Omega}}(e e, \alpha)=\nu_{A_{\Omega}}\left(\left(x x^{-1}\right)\left(x^{-1} x\right), \alpha\right)=\nu_{A_{\Omega}}\left(x\left(x^{-1} x^{-1}\right) x, \alpha\right) \\
\leq C\left(\nu_{A_{\Omega}}(x, \alpha), \nu_{A_{\Omega}}(x, \alpha)\right)=\nu_{A_{\Omega}}(x, \alpha) .
\end{gathered}
$$

Therefore, $\nu_{A_{\Omega}}(x, \alpha)=\nu_{A_{\Omega}}(e, \alpha)$. Hence $A_{\Omega}$ will be a constant function.

Proposition 3.13. Let $S$ be regular and $A_{\Omega}=\left(S \times \Omega, \mu_{A_{\Omega}}, \nu_{A_{\Omega}}\right)$ be an $\Omega$-bifuzzy set in $S$. If conditions (3) and (4) in Definition 3.7 are hold, then $A_{\Omega}$ will be an $\Omega$-bifuzzy bi-ideal of $S$ under norms ( $T$ and $C$ ).

Proof. Let $x, y \in S$ and $\alpha \in \Omega$. Since $S$ is regular, it follows that there exists $a \in S$, such that $y=y a y$, so that $\mu_{A_{\Omega}}(x y, \alpha)=\mu_{A_{\Omega}}(x(y a y) y, \alpha)=\mu_{A_{\Omega}}(x(y a) y, \alpha) \geq T\left(\mu_{A_{\Omega}}(x, \alpha), \mu_{A_{\Omega}}(y, \alpha)\right)$. Also $\nu_{A_{\Omega}}(x y, \alpha)=\nu_{A_{\Omega}}(x(y a y) y, \alpha)=\nu_{A_{\Omega}}(x(y a) y, \alpha) \leq C\left(\nu_{A_{\Omega}}(x, \alpha), \nu_{A_{\Omega}}(y, \alpha)\right)$. This completes the proof.

Proposition 3.14. Let $S$ be completely regular and $A_{\Omega}=\left(S \times \Omega, \mu_{A_{\Omega}}, \nu_{A_{\Omega}}\right)$ be an $\Omega$-bifuzzy bi-ideal of $S$ under norms ( $T$ and $C$ ). If $T$ and $C$ be idempotents, then $\mu_{A_{\Omega}}(x, \alpha)=\mu_{A_{\Omega}}\left(x^{2}, \alpha\right)$ and $\nu_{A_{\Omega}}(x, \alpha)=\nu_{A_{\Omega}}\left(x^{2}, \alpha\right)$ for all $x \in S$ and $\alpha \in \Omega$.

Proof. Let $x \in S$ and $\alpha \in \Omega$. Since $S$ is completely regular, so there exists $a \in S$ such that $x=x^{2} a x^{2}$. Then

$$
\begin{gathered}
\mu_{A_{\Omega}}(x, \alpha)=\mu_{A_{\Omega}}\left(x^{2} a x^{2}, \alpha\right) \geq T\left(\mu_{A_{\Omega}}\left(x^{2}, \alpha\right), \mu_{A_{\Omega}}\left(x^{2}, \alpha\right)\right) \\
=\mu_{A_{\Omega}}\left(x^{2}, \alpha\right) \geq T\left(\mu_{A_{\Omega}}(x, \alpha), \mu_{A_{\Omega}}(x, \alpha)\right)=\mu_{A_{\Omega}}(x, \alpha)
\end{gathered}
$$

and so $\mu_{A_{\Omega}}(x, \alpha)=\mu_{A_{\Omega}}\left(x^{2}, \alpha\right)$. Also

$$
\begin{gathered}
\nu_{A_{\Omega}}(x, \alpha)=\nu_{A_{\Omega}}\left(x^{2} a x^{2}, \alpha\right) \leq C\left(\nu_{A_{\Omega}}\left(x^{2}, \alpha\right), \nu_{A_{\Omega}}\left(x^{2}, \alpha\right)\right) \\
=\nu_{A_{\Omega}}\left(x^{2}, \alpha\right) \leq C\left(\nu_{A_{\Omega}}(x, \alpha), \nu_{A_{\Omega}}(x, \alpha)\right)=\nu_{A_{\Omega}}(x, \alpha)
\end{gathered}
$$

and then $\nu_{A_{\Omega}}(x, \alpha)=\nu_{A_{\Omega}}\left(x^{2}, \alpha\right)$. This completes the proof.

Theorem 3.15. Let $A_{\Omega}=\left(S \times \Omega, \mu_{A_{\Omega}}, \nu_{A_{\Omega}}\right)$ be an $\Omega$-bifuzzy bi-ideal of $S$ under norms ( $T$ and $C)$. If $S$ is a semilattice of groups, then the followings conditions hold.

(1) $\mu_{A_{\Omega}}(x, \alpha)=\mu_{A_{\Omega}}\left(x^{2}, \alpha\right)$ and $\nu_{A_{\Omega}}(x, \alpha)=\nu_{A_{\Omega}}\left(x^{2}, \alpha\right)$ for all $x \in S$ and $\alpha \in \Omega$.

(2) $\mu_{A_{\Omega}}(x y, \alpha)=\mu_{A_{\Omega}}(y x, \alpha)$ and $\nu_{A_{\Omega}}(x y, \alpha)=\nu_{A_{\Omega}}(y x, \alpha)$ for all $x, y \in S$ and $\alpha \in \Omega$. 
Proof. (1) If $S$ is a semilattice of groups, then $S$ is a union of groups and Lemma 2.16 gives that $S$ is completely regular. Now by Proposition 3.14 we have that $\mu_{A_{\Omega}}(x, \alpha)=\mu_{A_{\Omega}}\left(x^{2}, \alpha\right)$ and $\nu_{A_{\Omega}}(x, \alpha)=\nu_{A_{\Omega}}\left(x^{2}, \alpha\right)$ for all $x \in S$ and $\alpha \in \Omega$.

(2) Let $x, y \in S$ and $B[a]$ means the principal bi-ideal of $S$ generated by $a \in S$. By Lemma 2.17 we obtain $(x y)^{3}=(x y x)(y x y) \in B[x y x] B[y x y]=B[y x y](B[x y x])^{2} \subseteq B[y x y] S B[x y x] \subseteq$ $y x y S x y x \subseteq y x S y x$ for all $x, y \in S$. Then, there exists $a \in S$ such that $(x y)^{3}=(y x) a(y x)$. Similarly, we obtain $(y x)^{3}=(x y) a(x y)$. Now for any $\alpha \in \Omega$ we have $\mu_{A_{\Omega}}(x y, \alpha)=\mu_{A_{\Omega}}\left((x y)^{3}, \alpha\right)=$ $\mu_{A_{\Omega}}((y x) a(y x), \alpha) \geq T\left(\mu_{A_{\Omega}}(y x, \alpha), \mu_{A_{\Omega}}(y x, \alpha)\right)=\mu_{A_{\Omega}}(y x, \alpha)$.

Also

$$
\begin{gathered}
\nu_{A_{\Omega}}(x y, \alpha)=\nu_{A_{\Omega}}\left((x y)^{3}, \alpha\right)=\nu_{A_{\Omega}}((y x) a(y x), \alpha) \\
\leq C\left(\nu_{A_{\Omega}}(y x, \alpha), \nu_{A_{\Omega}}(y x, \alpha)\right)=\nu_{A_{\Omega}}(y x, \alpha) .
\end{gathered}
$$

Similarly, we have $\mu_{A_{\Omega}}(y x, \alpha) \geq \mu_{A_{\Omega}}(x y, \alpha)$ and $\nu_{A_{\Omega}}(y x, \alpha) \leq \nu_{A_{\Omega}}(x y, \alpha)$. This means that $\mu_{A_{\Omega}}(x y, \alpha)=\mu_{A_{\Omega}}(y x, \alpha)$ and $\nu_{A_{\Omega}}(x y, \alpha)=\nu_{A_{\Omega}}(y x, \alpha)$.

\section{Acknowledgements}

We would like to thank the reviewers for carefully reading the manuscript and making several helpful comments to increase the quality of the paper.

\section{References}

[1] Abbott, J. C. (1969). Sets, Lattices and Boolean Algebras, Allyn and Bacon, Boston.

[2] Atanassov, K. T. (1986). Intuitionistic fuzzy sets, Fuzzy Sets and Systems, 20 (1), 87-96.

[3] Atanassov, K. T. (1994). New operations defined over the intuitionistic fuzzy sets, Fuzzy Sets and Systems, 61, 137-142.

[4] Buckley, J. J. \& Eslami, E. (2002). An Introduction to Fuzzy Logic and Fuzzy Sets, SpringerVerlag Berlin Heidelberg GmbH.

[5] Dubois, D. \& Prade, H. (1988). Fuzzy Sets and Systems, Academic Press, New York.

[6] Gerstenkorn, T. \& Manko, J. (1995). Bifuzzy probabilistic sets, Fuzzy Sets and Systems, 71, 207-214.

[7] Hong, S. M., Jun, Y. B. \& Meng, J. (1995). Fuzzy interior ideals in semigroups, Indian J. Pure Appl. Math., 26(9), 859-863.

[8] Howie, J. (1995). Fundamentals of Semigroup Theory, London Mathematical Society Monographs. New Series, 12. Oxford Science Publications. The Clarendon Press, Oxford University Press, New York. 
[9] Hur, K., Jun, Y. B. \& Kim, H. S. (2005). $\Omega$-bifuzzy subsemigroups in semigroups, Honam Math. J., 27(1), 31-41.

[10] Klaua, D. (2010). An early approach toward graded identity and graded membership in set theory, Fuzzy Sets and Systems, 161, 2369-2379.

[11] Kuroki, N. (1982). Fuzzy semiprime ideals in semigroups, Fuzzy Sets and Systems, 8, 71-79.

[12] Kuroki, N. (1991). On fuzzy semigroups, Inform. Sci., 53, 203-236.

[13] Kuroki, N. (1992). Fuzzy generalized bi-ideals in semigroups, Inform. Sci., 66, 235-243.

[14] Lajos, S. (1972). On generalized bi-ideals in semigroups, Coll. Math. Soc. Janos Bolyai, Algebraic Theory of Semigroups, (G. Pollak, Ed.) North-Holland, 20, 335-340.

[15] Lajos, S. (1972). A note on semilattice of groups, Acta. Sci. Math. (Szeged), 33, 315-317.

[16] Liang, R., Lu, S., Wang, X. Lu, Y., Mandal, V., Patacsil, D. \& Kumar, D. (2006). A FuzzySet-Theory-Based Approach to Differential Gene Expression Data Analysis, BMC Bioinformatics, 7 (Suppl 4): S7.

[17] Mo, Z. W. \& Wang, X. P. (1993). On pointwise depiction of fuzzy regularity of semigroups, Inform. Sci., 74, 265-274.

[18] Petrich, M. (1973). Introduction to Semigroups, Columbus, Ohio.

[19] Rasuli, R. (2016). Fuzzy Ideals of Subtraction Semigroups with Respect to a $t$-norm and a t-conorm, The Journal of Fuzzy Mathematics Los Angeles, 24(4), 881-892.

[20] Rasuli, R. (2016). Fuzzy modules over a t-norm, Int. J. Open Problems Compt. Math., 9(3), $12-18$.

[21] Rasuli, R. (2016). Fuzzy Subrings over a t-norm, The Journal of Fuzzy Mathematics Los Angeles, 24(4), 995-1000.

[22] Rasuli, R. (2016). Norms over intuitionistic fuzzy subrings and ideals of a ring, Notes on Intuitionistic Fuzzy Sets, 22(5), 72-83.

[23] Samhan, M. A. (1993). Fuzzy congruences on semigroups, Inform. Sci., 74, 165-175.

[24] Wang, X. P. \& Liu, W. J. (1993). Fuzzy regular subsemigroups in semigroups, Inform. Sci., $68,225-231$.

[25] Zadeh, L. A. (1965). Fuzzy sets, Inform. Control., 8, 338-353. 